\title{
聚偏氟乙烯膜三维离子传输通道的构建及其 在全钒液流电池中的性能研究
}

\author{
王斐然蒋峰景* \\ (上海交通大学 机械与动力工程学院 上海 200240)
}

\begin{abstract}
摘要 随着新能源技术的不断发展, 大规模储能技术受到了广泛的关注. 全钒液流电池因其容量和功率设计灵活、安 全可靠、寿命长等优势成为发展较快的储能电池之一. 离子膜作为液流电池的关键部件, 对电池的能量转化效率、寿 命和成本具有显著影响. 本工作以高化学稳定性的聚偏氟乙烯作为膜材料, 利用聚乙二醇和聚乙烯吡咯烷酮分别作为 模板和稳定剂, 在聚偏氟乙烯膜内成功构建了具有较好 $\mathrm{H} / \mathrm{V}$ 离子选择性的三维离子传输通道. 电池性能测试表明, 该 聚偏氟乙烯(PVDF)离子膜有着出色的化学稳定性, 在 $100 \mathrm{~mA} \cdot \mathrm{cm}^{-2}$ 电流密度下, 具有 $98 \%$ 以上的电流效率和 $83.5 \%$ 的 能量效率. 此外, 聚偏氟乙烯具有价格低的显著特点, 使其在全钒液流电池中有较好的应用前景.
\end{abstract}

关键词＼cjkstart离子膜; 聚偏氟乙烯; 全钒液流电池; 大规模储能技术

\section{Construction of Three-dimensional Ion-conducting Channels of Poly(vinylidene fluoride) Membranes and Their Performance in Vanadium Redox Flow Battery}

\author{
Feiran Wang Fengjing Jiang* \\ (School of Mechanical Engineering, Shanghai Jiao Tong University, Shanghai 200240, China)
}

\begin{abstract}
With the increasing demand of renewable energy, large-scale energy storage technology has attracted extensive attention. Vanadium redox flow battery (VRFB), benefiting from its adjustable capacity, high safety and long life, has become one of the fastest developing batteries for large-scale energy storage. Ion exchange membrane is a key component of VRFB, which notably affects the efficiency, cost and stability of the batteries. However, as the most commonly used membrane in VRFBs, Nafion shows shortages of high vanadium permeability and high economic cost, which largely hinder the commercial application of VRFBs. In order to develop low-cost, durable and high-performance ion exchange membranes for flow batteries, in this work, dual-porous poly(vinylidene fluoride) ion exchange membranes have been developed, in which polyethylene glycol (PEG) and polyvinylidene pyrrolidone (PVP) are applied as the template and stabilizer molecules, respectively. As a result, three-dimensional ion-conducting network has been successfully built in the poly(vinylidene fluoride) (PVDF) matrix which enable fast proton conduction and ion selection. The ion-conducting channels and thereby the proton conductivity and $\mathrm{H} / \mathrm{V}$ ion selectivity of the membrane can be finely controlled by simply adjusting the PEG content in the casting solution. The unique dual porous structure of the membrane is clearly observed by using a scanning electron microscope. Battery efficiency including coulombic efficiency, voltage efficiency and energy efficiency have been characterized in VRFB single cells and the results show that the prepared PVDF ion-exchange membrane possesses high coulombic efficiency exceeding $98 \%$ and energy efficiency as high as $83.5 \%$ at a current density of $100 \mathrm{~mA} \cdot \mathrm{cm}^{-2}$, which are comparable to that of Nafion membranes. Moreover, the prepared PVDF ion-exchange membranes illustrate excellent chemical stability after the chemical stability test lasting for $30 \mathrm{~d}$. In a word, due to the very low cost of the polymer material, excellent chemical stability and good performance of the dual-porous PVDF membranes, the novel ion exchange membrane shows great prospect for the application in VRFBs.
\end{abstract}

Keywords ion-conducting membrane; poly(vinylidene fluoride); vanadium redox flow battery; large-scale energy storage technology

\section{1 引言}

在全球气候变暖, 化石能源存在短缺问题的大背景 下, 可再生能源, 如太阳能、风能、潮汐能、地热能等 新能源发电技术受到越来越多的关注并且在近年来迅 速发展进步 $[1]$. 随着技术的不断更新迭代, 新能源发电
在实际应用中出现受自然因素影响较大、发电能力不稳 定等问题逐渐显现 ${ }^{[2]}$. 全钒液流电池，由于其容量大、安 全性高、使用寿命长等优势, 可以作为大规模储能设备 起到削峰填谷、稳定发电功率输出的作用 ${ }^{[3-5]}$.

离子膜是全钒液流电池中重要的组成部件之一, 有

\footnotetext{
* E-mail: jfjzz@sjtu.edu.cn

Received May 24, 2021; published August 10, 2021.
} 
着分隔正负极电解液、导通质子、阻隔钒离子交叉污染 的作用 ${ }^{6-9]}$. 迄今为止, 商业化程度最高的电池隔膜是美 国杜邦公司研究开发的 Nafion 系列离子交换膜. Nafion 膜拥有较高的电导率和出色的化学稳定性, 然而 Nafion 膜的价格昂贵(约 $500 \sim 1000 \$ / \mathrm{m}^{2}$ )、钒离子渗透率高、 吸水溶胀等不足, 阻碍了其在全钒液流电池中的大规模 应用 ${ }^{[10-12]}$. 高性能、低成本、长寿命的全钒液流电池的 离子膜技术的开发对我国液流电池在储能领域的大规 模应用有重要的意义.

聚偏氟乙烯(PVDF)是一种半结晶非离子型疏水高 分子聚合物, 此前已有大量文献报道了其优异的机械强 度和极佳的耐腐蚀性 ${ }^{[13-14]}$. 因此 PVDF 被认为是较合适 用于制作全钒液流电池离子膜的高稳定性材料 [15-17]. 然 而, 由于 PVDF 本身具有较强的疏水性, 且聚合物本身 不具备传导离子的能力, 在离子膜制备和应用方面具有 较高的难度. 因此, 研究人员常用材料改性或构造传输 通道的方式制备 PVDF 离子膜. Mai 等 ${ }^{[18]}$ 将带有磺酸基 团的 Nafion 树脂与 PVDF 共混, PVDF 抑制了 Nafion 的 溶胀, 但当 PVDF 含量高于 $40 \%$ 时, 离子膜电阻较大、 电压效率较低. Ling 等 ${ }^{[19]}$ 制备的 $\mathrm{PVDF} / \mathrm{SiO}_{2}-\mathrm{SO}_{3} \mathrm{H}$ 复合 离子膜有着较低的钒离子渗透率, 在 $60 \mathrm{~mA} \cdot \mathrm{cm}^{-2}$ 电流 密度下能量效率为 $75.6 \%$. Luo 等 ${ }^{[20]}$ 在 PVDF 上接枝带 有磺酸基团的聚苯乙烯磺酸(PSSA), 电池能量效率能达 到 $75.8 \%$ 且循环 200 次保持稳定. $\mathrm{Li}$ 等 ${ }^{[21]}$ 利用 PVDF 半 结晶的特性, 通过温度和时间控制 PVDF 晶体结构制备 多孔的离子膜, 在电池测试中能稳定运行 650 次以上. Xue 等 ${ }^{[22]}$ 将预制 PVDF 薄膜浸没在溶剂/非溶剂混合溶 液中, 利用聚合物溶胀原理构造微孔离子膜, 在 100 $\mathrm{mA} \cdot \mathrm{cm}^{-2}$ 电流密度下能量效率达到 $78 \%$. 上述结果显示, 虽然基于 PVDF 的离子膜技术取得了较好的进展, 但是 仍有进一步提升性能的需求.

为了进一步提高 PVDF 离子膜在液流电池中的能量 效率, 本工作以聚乙二醇(PEG)作为模板分子、聚乙烯吡 咯烷酮(PVP)为稳定剂, 基于模板法制备了具有三维离 子传输通道的 PVDF 离子膜, 并研究了其在全钒液流电 池中的性能表现.

\section{2 结果与讨论}

\section{1 三维离子传输通道的构建}

作为非离子型可溶于水的模板分子, PEG 有着较高 的热稳定性和化学稳定性, 并且在常温下为液体, 能更 好地与铸膜液混合. 三维离子传输通道的构建过程如图 1 所示. PVP 因同时拥有亲水基团与疏水基团, 所以与 PVDF 和 PEG 都有着较好的相容性. 随着溶剂的挥发, 在聚合物基体中包裹着亲水模板分子 PEG, 并以一定的 排布方式稳定在疏水聚合物 PVDF 中, 从而在 PVDF 基 体中形成 PEG 连续相. 通过溶剂萃取模板的方法, 将溶 剂烘干的预制薄膜进行酸洗处理, PEG 被彻底去除之后,
连续相区构成了相互连通的三维离子传输通道. 通过改 变 PVP 和 PEG 的含量均能改变 PVDF 膜中的通道结构 和分布, 本工作将重点研究 PEG 含量对三维离子传输 通道形成的作用，及其对离子膜性能的影响.

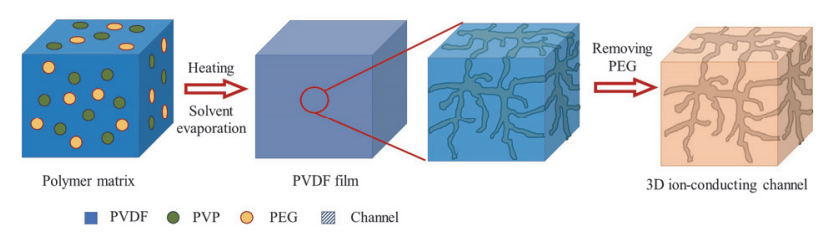

图 1 三维离子传输通道构建过程示意图

Figure 1 Schematic diagram of preparation of PVDF membrane with 3D ion-conducting channels

\subsection{PVDF 离子膜的微观形貌}

通过 SEM 可以清晰地观察到 PVDF 离子膜三维传 输通道的结构. 图 2 展示了 $\mathrm{PEG}$ 含量不同时, 离子膜上 表面的形貌特征. 由于相容性的影响, 液态的 PEG 分子 在聚合物基体中逐渐汇集而形成了液态的微区. 随着模 板分子 PEG 含量的增加, PEG 微区相互连通构成了三维 离子传输通道. 而当 PEG 含量达到 $25 \%$ 时, 除了形成大 量离子传导的微通道之外，还会在 PVDF 膜表面形成微 米级的 PEG 连续相. 宏观相分离的出现, 不利于获得高 离子选择性的离子膜. 因此, 在本体系中 PEG 造孔剂含 量应控制在 $25 \%$ 以下.
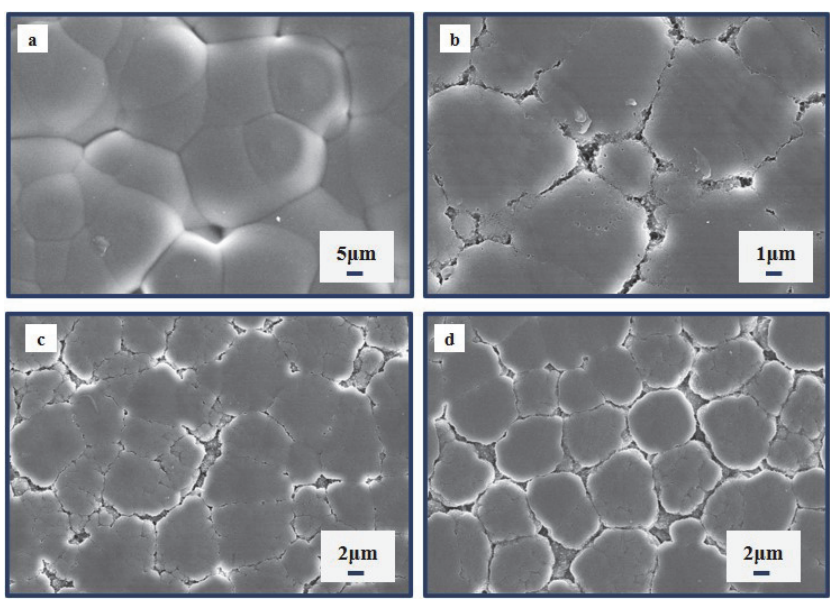

图 2 PVDF 离子膜(a) M-0, (b) M-15, (c) M-20 和(d) M-25 上表面扫描 电镜照片

Figure 2 SEM images of top view of the (a) M-0, (b) M-15, (c) M-20 and (d) M-25 membranes

图 3 展示了不同 PEG 含量时, 离子膜的断面微观形 貌照片. 随着 PEG 含量从 $0 \%$ 增加 $20 \%$, 离子膜中离子 传输通道变得丰富. 而当 PEG 含量达到 $25 \%$ 时, PVDF 膜中形成微米级的 PEG 连续相, 且离子膜内部结构变 得疏松, 不利于获得高机械强度的离子膜. 

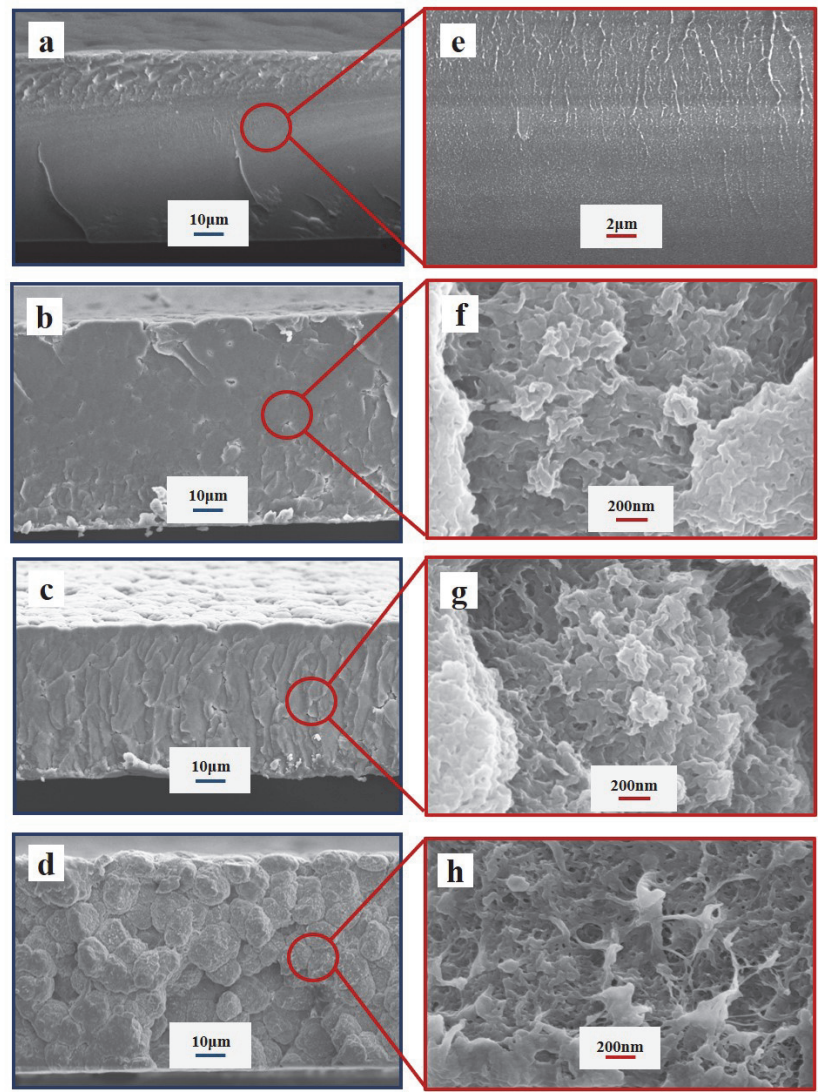

图 3 PVDF 离子膜(a, e) M-0, (b, f) M-15, (c, g) M-20 和(d, h) M-25 截 面扫描电镜图

Figure 3 SEM cross section images of (a, e) M-0, (b, f) M-15, (c, g) $\mathrm{M}-20$ and $(\mathrm{d}, \mathrm{h}) \mathrm{M}-25$ membranes

\subsection{PEG 含量对 PVDF 离子膜吸水率的影响}

PVDF 作为非离子型高分子聚合物, 因为没有亲水 基团的存在, 所以聚合物自身没有离子导电能力. 通过 以 PEG 作为造孔剂在聚合物基体中构建三维离子传输 通道, 制备 PVDF 离子膜. 图 4 展示的离子膜吸水率测 试的结果, 其中 $\mathrm{M}-0$ 的吸水率为 $8.4 \%$, 而 M-15、M-20 和 M-25 的吸水率分别为 $16.0 \% 、 24.3 \%$ 和 $36.9 \%$. 这也 证明, 当酸洗处理之后, 原本预制薄膜中 PEG 的连续相 区被水取代并填充, PEG 含量增加则在 PVDF 膜中三维 离子传输通道增多, 从而导致离子膜的吸水率增大.

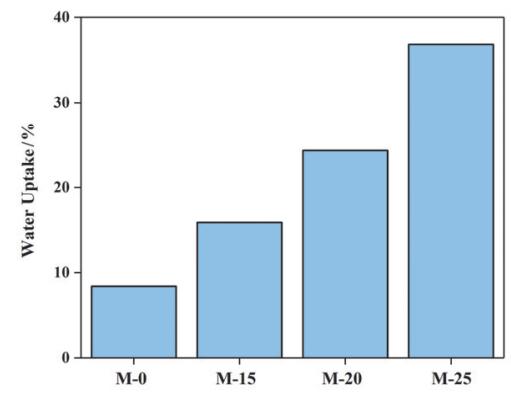

图 4 不同 PVDF 离子膜吸水率

Figure 4 Comparison of Water uptake of PVDF membranes

\subsection{PVDF 离子膜电导率与钒离子渗透率}

电导率反映离子膜传输质子的能力, 是离子膜的重 要性能指标. 电导率越高, 则传输质子的能力越强, 在 电池中表现出更高的电压效率. 如图 5 所示, M-15 的电 导率为 $11.7 \mathrm{mS} \bullet \mathrm{cm}^{-1}$, 而随着 $\mathrm{PEG}$ 含量越高的离子膜表 现出更高的电导率, M-20 和 M-25 的电导率分别上升到 $26.3 \mathrm{mS} \cdot \mathrm{cm}^{-1}$ 和 $46.7 \mathrm{mS} \cdot \mathrm{cm}^{-1}$. 这是因为随着造孔剂 PEG 的增加, 构建出更多的三维离子传输通道.

然而, 纳米尺度的三维离子传输通道在保证质子顺 利通过的同时会不可避免地传输一些不同价态的钒离 子. 根据文献可知 ${ }^{[23]}$, 水合离子半径由小到大依次为 $\mathrm{V}(\mathrm{II}) 、 \mathrm{~V}(\mathrm{IV}) 、 \mathrm{~V}(\mathrm{~V}) 、 \mathrm{~V}(\mathrm{III})$ ，而 $\mathrm{V}(\mathrm{II})$ 极易被空气氧化，因 此一般利用 $\mathrm{V}(\mathrm{IV})$ 来衡量离子膜的钒离子渗透率. 钒离 子渗透率升高, 会导致电解液交叉污染, 电池的库伦效 率降低. 图 5 所示的钒离子渗透率与电导率结果保持一 致, 离子传输通道越多, 钒离子渗透率也会相应的增加. 由于在水溶液中, 钒离子的 Stokes 半径和电荷密度比质 子大得多. 在膜内的迁移时, 钒离子和质子均是在 PVDF 纳米通道内的电解质水溶液中发生迁移. 由于三 维离子传输通道的 “体积排除效应” 以及钒离子和质子 的传导速率不同, 实现对钒离子和质子在膜内的选择性 透过. 然而 M-25 因为 PEG 含量较高, 除了纳米级微通 道外还形成了微米级传输通道, 而微米级的传输通道没 有选择分离氢离子和钒离子的能力, 因此 M-25 表现出 较高的钒离子渗透率 $\left(24.2 \times 10^{-7} \mathrm{~cm}^{2} \cdot \mathrm{min}^{-1}\right)$. 这一结果 与离子膜表面形貌表征结果保持一致. 根据实验可知, 自铸 Nafion 膜的钒离子渗透率为 $22.4 \times 10^{-7} \mathrm{~cm}^{2} \cdot \mathrm{min}^{-1}$, 而高钒离子渗透率会导致其在钒电池中应用时具有较 低的库伦效率. 综合电导率与钒离子渗透率的性能, $\mathrm{M}-20$ 离子膜拥有着较高的电导率 $\left(26.3 \mathrm{mS} \cdot \mathrm{cm}^{-1}\right)$ 和较低 的钒离子渗透率 $\left(13.2 \times 10^{-7} \mathrm{~cm}^{2} \cdot \mathrm{min}^{-1}\right)$, 是较为优选的 PVDF 离子膜.

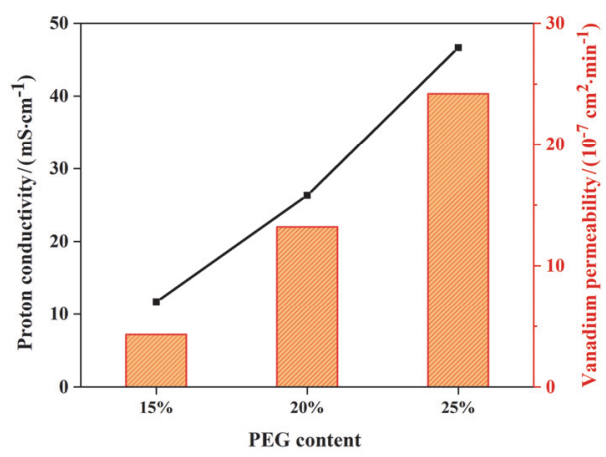

图 5 不同离子膜电导率与钒离子渗透率对比

Figure 5 Comparison of proton conductivity and vanadium permeability of PVDF membranes

\section{5 化学稳定性研究}

在全钒液流电池中, 离子膜被置于强氧化性、强酸 性的工作环境中, 这对于薄膜材料的化学稳定性提出了 
很高的要求.

图 6 展示了 PVDF 离子膜的化学稳定性测试结果. 根据图中可以看出, 当 M-20 没有进行酸洗处理之前, 离子膜中还存留着大量的 PEG. 因此, 在 V(V)强氧化性 的环境中这些物质被迅速的氧化, 测试溶液中 $\mathrm{V}(\mathrm{IV})$ 浓 度升高. 图 6(b)中展示了在进行 $30 \mathrm{~d}$ 原位化学稳定性测 试之后测试溶液的实物图. 浸泡 Nafion 膜与 M-20 膜的 测试溶液在 $30 \mathrm{~d}$ 后依然保持着 $\mathrm{V}(\mathrm{V})$ 溶液原有的亮黄色. 浸泡着 M-20 未经过酸洗处理的测试溶液已经变成了浅 绿色, 即亮黄色的 $V(V)$ 与蓝色的 $V(I V)$ 的混合色. 而单 纯加入 PEG 的测试溶液变成了蓝绿色, 即溶液中蓝色 的 $\mathrm{V}(\mathrm{IV})$ 更多. 为了进一步证实 M-20 膜的化学稳定性, 对于测试前后的 M-20 膜进行红外光谱分析. 图 6(c) 可 以看出经过 $30 \mathrm{~d}$ 原位化学稳定性测试之后, M-20膜的

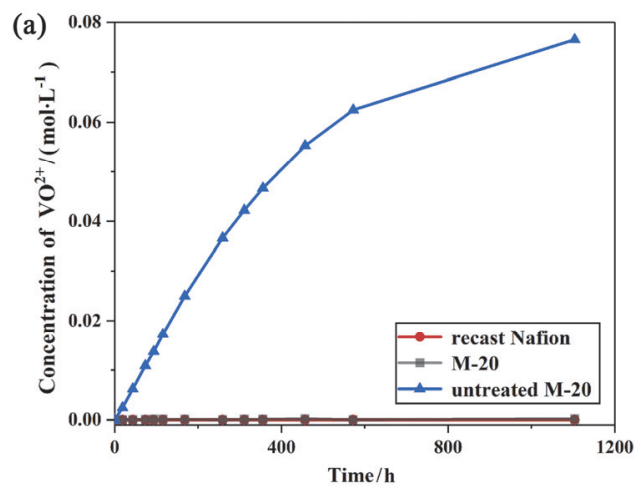

(b)
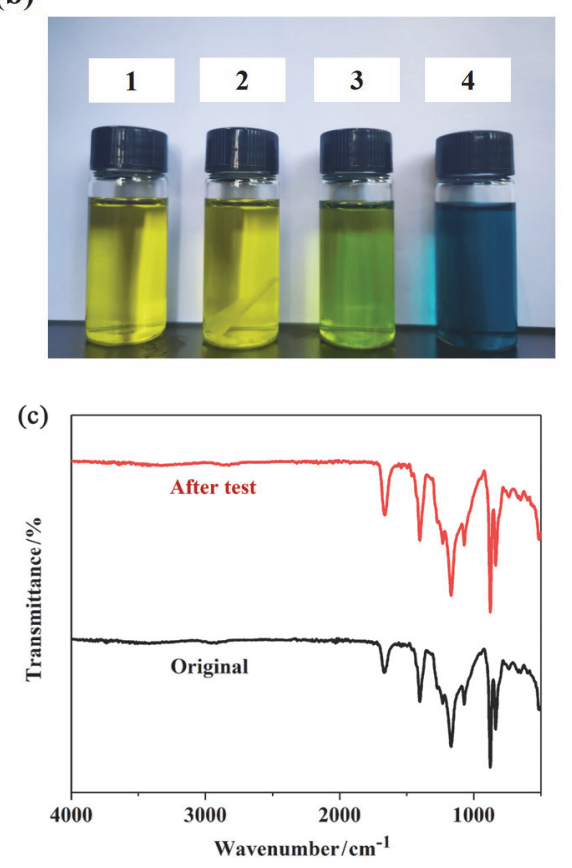

图 6 (a)在测试溶液中 V(IV)浓度的变化情况, (b)测试 $30 \mathrm{~d}$ 后溶液照 片 (1 为 Nafion 膜, 2 为 M-20, 3 为末进行酸洗处理的 M-20, 4 为纯 PEG), (c) M-20 化学稳定性测试前后红外光谱

Figure 6 (a) Concentration of V(IV) in testing solution, (b) picture of the testing solution after $30 \mathrm{~d}$ (1 Nafion, $2 \mathrm{M}-20,3$ untreated M-20, 4 Pure PEG), (c) FT-IR spectra of M-20
特征峰几乎完全一致, 即未检测到化学组分的变化. 研 究表明, Nafion 离子交换膜在全钒液流电池中具有足够 的耐腐蚀性和化学稳定性 ${ }^{[24]}$. 通过对比可知, 经过酸洗 处理之后的 M-20 离子膜亦具有较高的化学稳定性.

\section{6 单电池测试}

电池效率是衡量离子膜性能的重要参数, 主要由电 流效率、电压效率和能量效率组成. 单电池测试能够有 效地反映离子膜的综合性能, 是评价离子膜能否在液流 电池中实际应用的重要指标. M-20 膜的单电池测试不 同电流密度充放电曲线如图 7 所示. 随着电流密度的上 升, 电池的极化增强, 容量减小. 图 $8(\mathrm{a} \sim \mathrm{c})$ 可以看出,

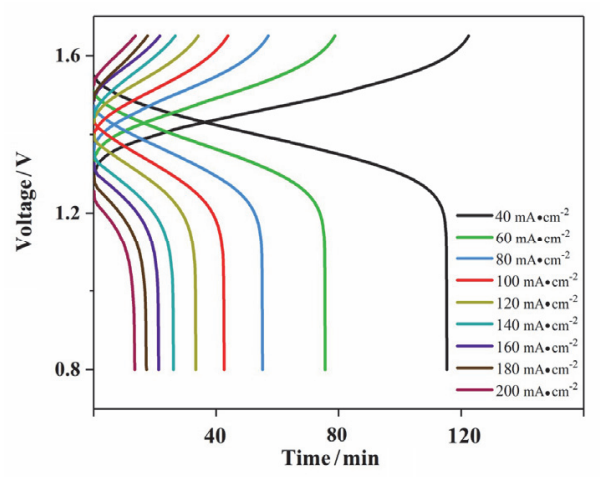

图 7 不同电流密度下 $\mathrm{M}-20$ 膜单电池测试充放电曲线

Figure 7 Charge-discharge curves of the single cell test of the M-20 membrane at different current densities
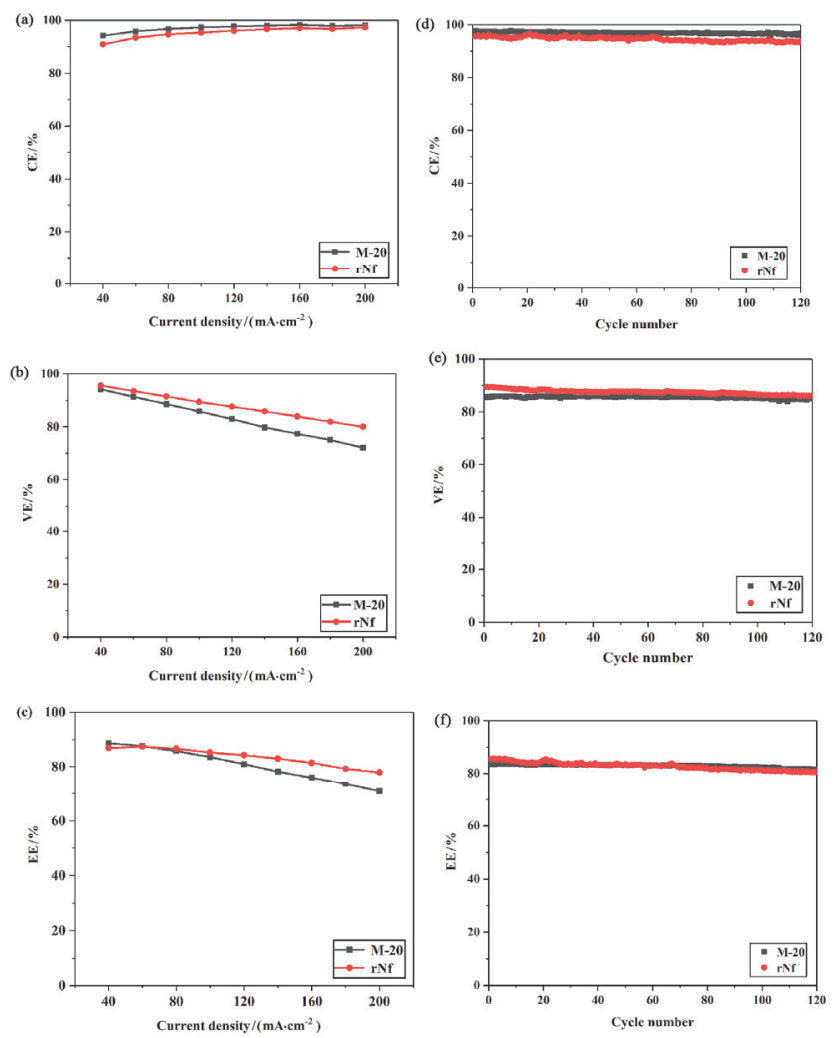

图 $8(\mathrm{a} \sim \mathrm{c})$ 不同电流密度充放电效率曲线, $(\mathrm{d} \sim \mathrm{f}) 100 \mathrm{~mA} \cdot \mathrm{cm}^{-2}$ 电流 密度下循环稳定性测试

Figure $8 \quad(a \sim c)$ Cell efficiency at various current densities and $(d \sim f)$ cycling test at $100 \mathrm{~mA} \cdot \mathrm{cm}^{-2}$ 
M-20 由于其较低的钒离子渗透率, 在电池测试中表现 出比自铸 Nafion 膜(rNf) 更高的库伦效率, 达到 $98 \%$ 以上 $\left(\mathrm{rNf}\right.$ 膜为 $96.1 \%$ ). 在 $100 \mathrm{~mA} \cdot \mathrm{cm}^{-2}$ 电流密度下, $\mathrm{M}-20$ 有 着 $83.5 \%$ 的能量效率，与 Nafion 膜 $85.3 \%$ 的能量效率较 为接近.

而循环测试可以更好地检测离子膜的工作稳定性, 是评估离子膜性能的重要指标. 图 $8(\mathrm{~d} \sim \mathrm{f})$ 展示了在 100 $\mathrm{mA} \cdot \mathrm{cm}^{-2}$ 电流密度下电池 120 次循环充放电的效率. 自 铸 Nafion 膜(rNf)因为在电解液中浸泡发生了溶胀, 导 致电流效率出现了下降, 而 M-20 在电池循环测试中保 持着稳定的性能, 库伦效率与能量效率分别稳定在 96\%、82\%以上. 循环测试表明 M-20 在全钒液流电池中 具有较好的效率和循环稳定性.

\section{3 结论}

本工作以非离子型聚合物 PVDF 作为基体聚合物, 利用模板法制备了具有三维离子传输通道的离子膜. PVDF 离子膜拥有出色的化学稳定性, 在电池测试中表 现出能与 Nafion 膜相妶美的高库伦效率和能量效率 $(98 \%, 83.5 \%)$, 并且在 $100 \mathrm{~mA} \cdot \mathrm{cm}^{-2}$ 电流密度下循环 120 次仍保持着稳定的电池效率. 预计用该方法制备的 PVDF 离子膜成本可降至 $50 \$ / \mathrm{m}^{2}$ 以下, 仅为商用 Nafion 膜的 1/10 左右. 研究结果表明, 制备的具有三维离子传 输通道的 PVDF 离子膜在全钒液流电池中具有较好的应 用前景.

\section{4 实验部分}

\section{1 实验原材料}

聚偏氟乙烯粉末 HSV 900, 法国阿科玛公司; 聚乙 烯吡咯烷酮 K60 (PVP), 上海泰坦科技股份有限公司; $N, N$-二甲基甲酰胺(DMF)、聚乙二醇 400 (PEG400), 上 海凌峰化学试剂有限公司, 分析纯.

\section{2 膜的制备}

按一定的质量比, 将 PVDF、PVP、PEG 溶解于 DMF 中, 在真空条件下室温静置过夜脱泡, 得到澄清均匀的 铸膜液. 其中 PVP 含量设定为 PVDF 质量的 $33 \%$. 将铸 膜液流延至洁净的玻璃板上, 放入烘箱 $80{ }^{\circ} \mathrm{C}$ 加热 $7 \mathrm{~h}$ 以去除溶剂，得到预制薄膜. 将预制薄膜浸没于 1 $\mathrm{mol} / \mathrm{L}$ 硫酸溶液中在 $80{ }^{\circ} \mathrm{C}$ 条件下加热处理 $1 \mathrm{~h}$ 以去除模 板. 最后将薄膜浸泡在去离子水中进行反复清洗, 最终 将制得 PVDF 离子膜. 在本文讨论中, 铸膜液与空气接 触面设为薄膜上表面, 与玻璃板接触面设为下表面. 为 了方便表述, 将不同模板含量的薄膜表示为 $\mathrm{M}-x$, 其中 $x$ 为 PEG 在总聚合物中的含量.

\section{3 膜的性能测试和表征}

(1)表面形貌表征

将薄膜在液氮中进行淬断，再在表面喷金 $40 \mathrm{~s}$, 然
后用日本日立公司场发射扫描电子显微镜(S-4800)观察 膜的上表面与截面形貌.

(2)吸水率测试

将薄膜放入烘箱中 $80{ }^{\circ} \mathrm{C}$ 真空干燥 $6 \mathrm{~h}$, 称得薄膜干 重 $\left(m_{\mathrm{d}}\right)$. 再将薄膜浸没在去离子水中 $3 \mathrm{~d}$ 后, 取出薄膜并 用滤纸擦拭表面多余水分, 记录得到薄膜的湿重 $\left(m_{\mathrm{w}}\right)$, 并利用公式(1)计算出薄膜的吸水率.

$$
\text { 含水率 }=\frac{m_{\mathrm{w}}-m_{\mathrm{d}}}{m_{\mathrm{d}}} \times 100 \%
$$

(3)电导率、钒离子渗透率测试

在英国输力强公司生产的电化学工作站上利用交 流阻抗法测试得到薄膜电阻, 并计算得到薄膜的电导率 $(\sigma)$, 测试溶液为 $3 \mathrm{~mol} / \mathrm{L}$ 的硫酸. 将薄膜用球形接口夹 夹持在两个储液罐中间, 左侧储液罐内为 $1.5 \mathrm{~mol} / \mathrm{L}$ $\mathrm{VOSO}_{4}$ 与 $3 \mathrm{~mol} / \mathrm{L} \mathrm{H}_{2} \mathrm{SO}_{4}$ 混合溶液, 右侧储液罐内为 1.5 $\mathrm{mol} / \mathrm{L} \mathrm{MgSO}_{4}$ 与 $3 \mathrm{~mol} / \mathrm{L} \mathrm{H}_{2} \mathrm{SO}_{4}$ 混合溶液 ${ }^{[25]}$. 一定时间 间隔取右侧储液罐中的溶液利用紫外光分光光度计(测 试波长 $762 \mathrm{~nm}$ )测量溶液吸光度, 计算得到薄膜钒离子 渗透率 $(P)$.

$$
\begin{aligned}
\sigma & =\frac{L}{S \times R} \\
P & =\frac{V_{\mathrm{B}} L}{A \times\left(c_{\mathrm{A}}-c_{\mathrm{B}}(t)\right)} \cdot \frac{\mathrm{d} c_{\mathrm{B}}(t)}{\mathrm{d} t}
\end{aligned}
$$

其中, $L$ 为薄膜厚度, $S 、 A$ 分别为薄膜测试电导率和钒离 子渗透率的有效面积, $R$ 为薄膜电阻, $V_{\mathrm{B}}$ 为右侧储液罐 内溶液体积, $c_{\mathrm{A}}$ 和 $c_{\mathrm{B}}(t)$ 分别为左侧和右侧储液罐内钒离 子浓度.

\section{(4)化学稳定性测试}

将薄膜浸泡在 $0.15 \mathrm{~mol} / \mathrm{L}$ 的 $\left(\mathrm{VO}_{2}\right)_{2} \mathrm{SO}_{4}$ 和 $3 \mathrm{~mol} / \mathrm{L}$ $\mathrm{H}_{2} \mathrm{SO}_{4}$ 的混合溶液中, 在烘箱中 $40{ }^{\circ} \mathrm{C}$ 保温, 一定时间 间隔取部分溶液利用紫外光分光光度计(测试波长 762 $\mathrm{nm})$ 测量溶液吸光度 ${ }^{[26]}$. 溶液吸光度越高, 则更多的 $\mathrm{V}(\mathrm{V})$ 被还原成为 $\mathrm{V}(\mathrm{IV})$, 薄膜化学稳定性越低.

(5)红外光谱分析

将待测薄膜放入烘箱中, 真空条件下 $80{ }^{\circ} \mathrm{C}$ 干燥 $8 \mathrm{~h}$ 得到待测样品. 利用红外光谱仪 Nicolet 6700 对于样品 进行红外光谱分析.

(6)单电池测试

全钒液流电池的单电池结构如图 9 所示. 正极电解 液为 $1.5 \mathrm{~mol} / \mathrm{L} \mathrm{V}(\mathrm{V}) / \mathrm{V}(\mathrm{IV})$ 和 $3 \mathrm{~mol} / \mathrm{L} \mathrm{H}_{2} \mathrm{SO}_{4}$ 混合溶液, 负极电解液为 $1.5 \mathrm{~mol} / \mathrm{L} \mathrm{V}(\mathrm{II}) / \mathrm{V}(\mathrm{III})$ 和 $3 \mathrm{~mol} / \mathrm{L} \mathrm{H}_{2} \mathrm{SO}_{4}$ 混 合溶液, 且正负极电解液体积均为 $50 \mathrm{~mL}$, 电池中电解 液通过蠕动洜进行循环. 电池充放电截止电压分别为 $1.65 \mathrm{~V}$ 和 $0.80 \mathrm{~V}$. 不同电流密度充放电测试范围为 $40 \sim$ $200 \mathrm{~mA} \cdot \mathrm{cm}^{-2}$, 循环测试电流密度为 $100 \mathrm{~mA} \cdot \mathrm{cm}^{-2}$. 单电 池的测试在氮气环境下进行, 工作温度为 $25{ }^{\circ} \mathrm{C}$. 电池 的库伦效率 $(\mathrm{CE})$ 、电压效率 $(\mathrm{VE})$ 和能量效率 $(\mathrm{EE})$ 计算公 式如下. 


$$
\begin{aligned}
& C E=\frac{\int I_{\mathrm{d}} \mathrm{d} t}{\int I_{\mathrm{c}} \mathrm{d} t} \times 100 \% \\
& V E=\frac{\int V_{\mathrm{d}} I_{\mathrm{d}} \mathrm{d} t}{\int V_{\mathrm{c}} I_{\mathrm{c}} \mathrm{d} t} \times 100 \% \\
& E E=\frac{C E}{V E} \times 100 \%
\end{aligned}
$$

其中, $I_{\mathrm{c}}, I_{\mathrm{d}}$ 分别表示充电、放电电流, $V_{\mathrm{c}}, V_{\mathrm{d}}$ 分别表示充 电、放电电压.

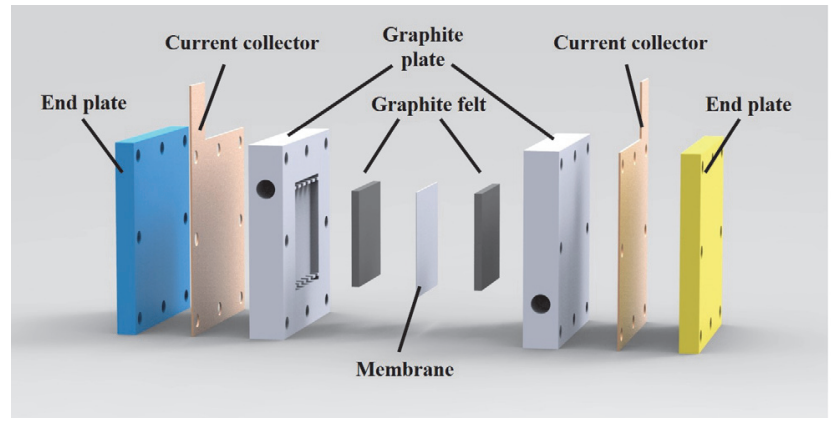

图 9 全钒液流电池单电池结构

Figure 9 Structure of the VRFB single cell

\section{References}

[1] Turner, J. A. Science 1999, 285, 687

[2] Qing, G. L.-T.; Guo, W.-N.; Liu, P.; Wang, B.-G. J. Electrochem. $\mathbf{2 0 1 5}, 21,449$ (in Chinese). (青格勒图, 郭伟男, 刘平, 王保国, 电 化学, 2015, 21, 449.)

[3] Kim, S.; Yan, J.; Schwenzer, B.; Zhang, J.; Li, L.; Liu, J.; Yang, Z.; Hickner, M. A. Electrochem. Commun. 2010, 12, 1650.

[4] Zhao, Y.-T.; Xi, J.-Y.; Teng, X.-G.; Wu, Z.-H.; Qiu, X.-P. Acta Chim. Sinica 2011, 69, 132 (in Chinese). (赵永涛, 席靖宇, 腾祥国, 武增 华, 邱新平, 化学学报, 2011, 69, 132.)
[5] Li, L.; Kim, S.; Wang, W.; Vijayakumar, M.; Nie, Z.; Chen, B.; Zhang, J.; Xia, G.; Hu, J.; Graff, G. Adv. Energy Mater. 2011, 1, 394.

[6] Ulaganathan, M.; Aravindan, V.; Yan, Q.; Madhavi, S.; Skyllas-Kazacos, M.; Lim, T. M. Adv. Energy Mater. 2016, 3 , 1500309.

[7] Wei, X.; Nie, Z.; Luo, Q.; Li, B.; Chen, B.; Simmons, K.; Sprenkle, V.; Wei, W. Adv. Energy Mater. 2014, 3, 1215.

[8] Wan, Y. H.; Sun, J.; Jiang, H. R.; Fan, X. Z.; Zhao, T. S. J. Power Sources 2021, 489, 229502.

[9] Wang, F.-R.; Jiang, F.-J. Prog. Chem. 2021, 33, 462 (in Chinese). (王斐然, 蒋峰景, 化学进展, 2021, 33, 462.)

[10] Zhou, X.; Xue, R.; Zhong, Y.; Zhang, Y.; Jiang, F. J. Membrane Sci. 2020, 595, 117614.

[11] Zhang, B.-G.; Zhang, S.-H.; Xing, D.-B.; Yin, C.-X.; Han, R.-L.; Jian, X.-G. Acta Chim. Sinica 2011, 69, 2583 (in Chinese). (张本贵, 张守海, 邢东博, 尹春香, 韩润林, 赛锡高, 化学学报, 2011, 69 , 2583.)

[12] Li, L.-Q.; Chen, J.-W.; Lu, H.; Jiang, C.-P.; Gao, S.; Yang, X.; Lian, X.-J.; Liu, X.-J.; Wang, R.-L. Acta Chim. Sinica 2009, 67, 2785 (in Chinese). (李良琼，陈金伟，鲁惠，姜春萍，高山，杨金鍂，练晓娟， 刘效疆, 王瑞林, 化学学报, 2009, 67, 2785.)

[13] Ling, L. A.; Min, X. A.; Dh, B.; Shan, R. A.; Sw, A.; Ym, A. J. Membrane Sci. 2019, 585, 230.

[14] Fu, L.; Hashim, N. A.; Liu, Y.; Abed, M.; Li, K. Fuel Energ. Abstr. 2011, 375, 1 .

[15] Li, B.-Y.; Liu, Z.-H.; Wang, B.-G. CIESC J. 2017, 68, 732 (in Chinese). (李冰洋, 刘珍豪, 主保国, 化工学报, 2017, 68,732 .)

[16] Yang, X.; Zhu, H.; Jiang, F.; Zhou, X. J. Power Sources 2020, 473, 228586.

[17] Lai, Y.; Wan, L.; Wang, B. Membranes 2019, 9, 89.

[18] Mai, Z.; Zhang, H.; Li, X.; Xiao, S.; Zhang, H. J. Power Sources 2011, 196, 5737.

[19] Ling, L.; Xiao, M.; Han, D.; Ren, S.; Meng, Y. J. Membrane Sci. 2019, 585, 230

[20] Luo, X.; Lu, Z.; Xi, J.; Wu, Z.; Zhu, W.; Chen, L.; Qiu, X. J. Phys. Chem. B 2005, 109, 20310.

[21] Li, B.; Wang, B.; Liu, Z.; Qing, G. J. Membrane Sci. 2016, 111

[22] Xue, R.; Jiang, F.; Wang, F.; Zhou, X. J. Power Sources 2019, 449, 227475.

[23] Sun, C.; Jian, C.; Zhang, H.; Xi, H.; Luo, Q. J. Power Sources 2010, $195,890$.

[24] Jiang, B.; Wu, L.; Yu, L.; Qiu, X.; Xi, J. J. Membrane Sci. 2016, 510,18

[25] Zhang, Y.; Zhou, X.; Xue, R.; Yu, Q.; Jiang, F.; Zhong, Y. Int. J. Hydrogen Energ. 2019, 44, 5997.

[26] Zhao, Y.; Zhang, H.; Xiao, C.; Lin, Q.; Li, X. Nano Energy 2018, 48, 353.

(Cheng, B.) 